\title{
The Influence of the Aspect Ratio on the Composite Material Bridge Deck Structures
}

\author{
Bong-Koo Han ${ }^{\dagger}$
}

\begin{abstract}
Theories for composite material structures are too difficult for such design engineers for construction and some simple but accurate enough methods are necessary. The author has reported that some laminate orientations have decreasing values of $D_{16}, B_{16}, D_{26}$ and $B_{26}$ stiffnesses as the ply number increases. For such plates, the fiber orientations given above behave as specially orthotropic plates and simple formulas developed by the author. Most of the bridge deck structures on girders have large aspect ratios. For such cases further simplification is possible by neglecting the effect of the longitudinal moment terms $M_{x}$ on the relevant partial differential equations of equilibrium. In this paper, the result of the study on the subject problem is presented.
\end{abstract}

Key Words: composite material structures, specially orthotropic, longitudinal moment terms, finite difference method, beam theory

\section{INTRODUCTION}

The future of material industry will depend on if and when the conventional construction materials are replaced by composite materials. If composite materials are used for construction, the quantity is huge : in tons, not in kilos or pounds. A composite materials can be used economically and efficiently in broad civil engineering applications when standards and processes for analysis, design, fabrication, construction and quality control are established. The problem of deteriorating infrastructures is very serious all over the world. The U.S. Civil Engineering Research Foundation (CERF) report, "HighPerformance Construction Material and System : An Essential Program for America and its Infrastructure", published, in collaboration with several organizations, U.S. Department of Transportation figures as follows:

(1) The road bridge condition in U.S.A at the year 2009, 149,654 of the Americans 603,259 bridges are structurally deficient or obsolete. (structurally deficient 71,177 , functionally obsolete 78,477 )

(2) 199,584 of these bridges are more than 50 years old and unsuitable for current or projected traffic.

(3) Traffic delays alone will cost Americans $\$ 115$ billion per year in lost work time and fuel by the year 2009.

Steel girders become rusty. The reinforcing bars embedded in concrete beams or slabs are subject to corrosion caused by electro-chemical action. Underground fuel thanks are under similar condition. In 1979, the U.S. Bureau of Standards (NIST) study showed that yearly loss caused by corrosion related damages mounted to 82 billion dollars, about $4.9 \%$ of GNP. About 32 billion dollars could be saved if existing technologies were used to prevent such losses [1].

These figures are in the United States of America, where various federal, state, and other agencies are doing their best in maintaining such structures in good condition. The issue of deteriorating and damaged infrastructures and lifelines has become a critically important subject in the United States as well as Japan and Europe. The problem in developing nations, where degree of construction quality control and maintenance are in question, must be much more profound $[1,2]$.

The composite materials can be effectively used for repairing such structures. Because of the advantages of these materials, such repair job can fulfill two purposes:

(1) Repair of existing damage caused by corrosion, impact, earthquake, and others.

(2) Reinforcing the structure against anticipated future sit- 
uation which will require increasing the load beyond the design parameters used for this structure.

Before making any decision on repair work, reliable non destructive evaluation is necessary. One of the dependable methods is to evaluate the in-situ stiffness of the structure by means of obtaining the natural frequency. By comparing the in-situ stiffness with the one obtained at the design stage, the degree of damage can be estimated rather accurately.

The reinforced concrete slab can be assumed as a $[0,90,0]_{\mathrm{r}}$ type specially orthotropic plate as a close approximation, assuming that the influence of $B_{16}, B_{26}, D_{16}$, and $D_{26}$ stiffness are negligible. Many of the bridge and building floor systems, including the girders and cross beams, also behave as similar specially orthotropic plates. Such plates are subject to the concentrated mass/masses in the form of traffic loads, or the test equipments such as the accelerator in addition to their own masses. Analysis of such problems is usually very difficult.

The most of the design engineers for construction has academic background of bachelors degree. Theories for composite structures are too difficult for such engineers and some simple but accurate enough methods are necessary.

The author has reported that some laminate orientations such as $[\alpha, \beta]_{r},[\alpha, \beta, \gamma]_{r},[\alpha, \beta, \beta, \alpha, \alpha, \beta]_{r}$ and $[\alpha, \beta, \beta, \gamma, \alpha, \alpha$, $\beta]_{r}$ with $\alpha=-\beta$, and $\gamma=0^{\circ}$ or $90^{\circ}$ and with increasing $r$, have decreasing values of $B_{16}, B_{26}, D_{16}$, and $D_{26}$ stiffness. Most of the civil and architectural structures are large in sizes and the numbers of laminae are large, even though the thickness to length ratios are small enough to allow to neglect the transverse shear deformation effects in stress analysis. For such plates, the fiber orientations given above behave as specially orthotropic plates and simple formulas developed by the reference $[1,3]$ can be used. Most of the bridge and building slabs on girders have large aspect ratios. For such cases further simplification is possible by neglecting the effect of the longitudinal moment terms $\left(M_{x}\right)$ on the relevant partial differential equations of equilibrium [3]. In this paper, the result of the study on the subject problem is presented. Even with such assumption, the specially orthotropic plate with boundary conditions other than Navier or Levy solution types, or with irregular cross section, or with nonuniform mass including point masses, analytical solution is very difficult to obtain. Numerical method for eigenvalue problems are also very much involved in seeking such a solution [4-7].

The method of vibration analysis used is the one developed by the author. He developed and reported, in 1974 [8], a simple but exact method of calculating the natural frequency of beam and tower structures with irregular cross sections and attached mass/masses. Since 1989, this method has been extended to two dimensional problems with several types of given conditions and has been reported at several international conferences [7]. This method uses the deflection influence surfaces. The finite difference method (F.D.M) is used for this purpose, in this paper.

\section{METHOD OF ANALYSIS}

The equilibrium equation for the specially orthotropic plate is:

$$
D_{1} \frac{\partial^{4} w}{\partial x^{4}}+2 D_{3} \frac{\partial^{4} w}{\partial x^{2} \partial y^{2}}+D_{2} \frac{\partial^{4} w}{\partial y^{4}}=q(x, y)
$$

where $D_{1}=D_{11}, D_{2}=D_{22}, D_{3}=D_{12}+2 D_{66}$

The assumptions needed for this equation are:

(1) The transverse shear deformation is neglected.

(2) Specially orthotropic layers are arranged so that no coupling terms exist, i.e, $B_{i j}=0, D_{16}=D_{26}=0$.

(3) No temperature or hygrothermal terms exist.

The purpose of this paper is to demonstrate, to the practicing engineers, how to apply this equation to the slab systems made of plate girders and cross beams.

In case of an orthotropic plate with boundary conditions other than Navier or Levy solution type, or with irregular cross section, or with nonuniform mass including point masses, analytical solution is very difficult to obtain. Numerical methods for eigenvalue problems are also very much involved in seeking such a solution [9-12]. Finite difference method is used in this paper. The resulting linear algebraic equations can be used for any cases with minor modifications at the boundaries, and so on.

The problem of deteriorating infrastructures is very serious all over the world. Before making any decision on repair work, reliable non destructive evaluation is necessary. One of the dependable methods is to evaluate the in-situ stiffness of the structure by means of obtaining the natural frequency. By comparing the in-situ stiffness with the one obtained at the design stage, the degree of damage can be estimated rather accurately.

The basic concept of the Rayleigh method, the most popular analytical method for vibration analysis of a single degree of freedom system, is the principle of conservation of energy; the energy in a free vibrating system must remain constant if no damping forces act to absorb it. In case of a beam, which has an infinite number of degree of freedom, it is necessary to assume a shape function in order to reduce the beam to a single degree of freedom system. The frequency of vibration can be found by equating the maximum strain energy developed during the motion to the maximum kinetic energy. This method, however, yields the solution either equal to or larger than the real one. Recall that Rayleigh's quotient $\geq 1[15$, pp 189-191]. For a complex beam, assuming a correct shape function is not possible. In such cases, the solution obtained is larger than the real one.

Design engineers need to calculate the natural frequencies of such element but obtaining exact solution to such problems is very much difficult. Pretlove reported a method of analysis of beams with attached masses using the concept of effective mass. This method, however, is useful only for certain simple types of beams. Such problems can be easily solved by pre- 
sented method.

A simple but exact method of calculating the natural frequency corresponding to the first mode of vibration of beam and tower structures with irregular cross sections and attached mass/masses was developed and reported by Kim in 1974 [8]. This method consists of determining the deflected mode shape of the member due to the inertia force under resonance condition. Beginning with initially "guessed" mode shape, "exact" mode shape is obtained by the process similar to iteration. Recently, this method was extended to two dimensional problems including composite laminates, and has been applied to composite plates with various boundary conditions with/ without shear deformation effects and reported at several international conferences including the Eighth Structures Congress (1990) and Fourth Materials Congress (1996) of American Society of Civil Engineers.

This method is used for vibration analysis in this paper. A natural frequency of a structure is the frequency under which the deflected mode shape corresponding to this frequency begins to diverge under the resonance condition. From the deflection caused by the free vibration, the force required to make this deflection can be found, and from this force, resulting deflection can be obtained. If the mode shape as determined by the series of this process is sufficiently accurate, then the relative deflections (maximum) of both the converged and the previous one should remain unchanged under the inertia force related with this natural frequency. Vibration of a structure is a harmonic motion and the amplitude may contain a part expressed by a trigonometric function.

\subsection{Vibration analysis}

In this paper, the method of analysis given in detail, in the reference book is briefly repeated [2].

The magnitudes of the maximum deflection at a certain number of points are arbitrarily given as

$$
w(i, j)^{(1)}=W(i, j)^{(1)}
$$

where $(i, j)$ denotes the point under consideration. This is absolutely arbitrary but educated guessing is good for accelerating convergence. The dynamic force corresponding to this (maximum) amplitude is

$$
F(i, j)^{(1)}=m(i, j)\left[w(i, j)^{(1)}\right]^{2} w(i, j)^{(1)}
$$

The "new" deflection caused by this force is a function $F$ and can be expressed as

$$
\begin{aligned}
w(i, j)^{(2)} & =f\left\{m(i, j)\left[w(i, j)^{(1)}\right]^{(2)} w(i, j)^{(1)}\right\} \\
& =\sum^{k, l} \Delta(i, j, k, l)\left\{m(i, j)\left[w(i, j)^{(1)}\right]^{(2)} w(k, l)^{(1)}\right\}
\end{aligned}
$$

where $\Delta(i, j, k, l)$ is the deflection influence surface. The relative (maximum) deflections at each point under consideration of a structural member under resonance condition, $w(i, j)^{(1)}$ and $w(i, j){ }^{(2)}$, have to remain unchanged and the following condition has to be held:

$$
w(i, j)^{(1)} / w(i, j)^{(2)}=1
$$

From this equation, $w(i, j)^{(1)}$ at each point of $(i, j)$ can be obtained. But they are not equal in most cases. Since the natural frequency of a structural member has to be equal at all points of the member, i.e. $w(i, j)$ should be equal for all $(i, j)$, this step is repeated until sufficient equal magnitude of $w(i, j)$ is obtained at all $(i, j)$ points.

However, in most cases, the difference between the maximum and the minimum values of $w(i, j)$ obtained by the first cycle of calculation is sufficiently negligible for engineering purposes. The accuracy can be improved by simply taking the average of the maximum and the minimum, or by taking the value of $w(i, j)$ where the deflection is the maximum. For the second cycle, $w(i, j)^{(3)}$ is

$$
w(i, j)^{(3)}=f\left\{m(i, j)\left[w(i, j)^{(2)}\right]^{2} w(i, j)^{(2)}\right\}
$$

the absolute numerics of $w(i, j)^{(2)}$ can be used for convenience.

\subsection{Finite difference method}

The method used in this paper requires the deflection influence surfaces. F.D.M is applied to the governing equation of the specially orthotropic plates,

$$
\begin{aligned}
& D_{1} \frac{\partial^{4} w}{\partial x^{4}}+2 D_{3} \frac{\partial^{4} w}{\partial x^{2} \partial y^{2}}+D_{2} \frac{\partial^{4} w}{\partial y^{4}} \\
& =q(x, y)-k w+N x \frac{\partial^{2} w}{\partial x^{2}}+N y \frac{\partial^{2} w}{\partial y^{2}}+2 N x y \frac{\partial^{2} w}{\partial x \partial y}
\end{aligned}
$$

where, $D_{1}=D_{11}, D_{2}=D_{22}, D_{3}=\left(D_{12}+2 D_{66}\right)$

The number of the pivotal points required in the case of the order of error $\Delta_{2}$, where $\Delta$ is the mesh size, is five for the central differences.

This makes the procedure at the boundaries complicated. In order to solve such problem, the three simultaneous partial differential equations of equilibrium with three dependent variables, $w, M_{x}$ and $M_{y}$, are used instead of Eq. (7) with $N_{x}=N_{y}=N_{x y}=0 \quad[5,7]$.

$$
\begin{aligned}
& D_{1} \frac{\partial^{2} M x}{\partial x^{2}}-4 D_{66} \frac{\partial^{4} w}{\partial x^{2} \partial y^{2}}+\frac{\partial^{2} M y}{\partial y^{2}}=-q(x, y)+k w(x, y) \\
& M_{x}=-D_{11} \frac{\partial^{2} w}{\partial x^{2}}-D_{12} \frac{\partial^{2} w}{\partial y^{2}} \\
& M_{y}=-D_{12} \frac{\partial^{2} w}{\partial x^{2}}-D_{22} \frac{\partial^{2} w}{\partial y^{2}}
\end{aligned}
$$


If F.D.M. is applied to these equations, the resulting matrix equation is very large in sizes, but the tridiagonal matrix calculation scheme used by Kim is very efficient to solve such equations [3].

In order to confirm the accuracy of the F.D.M., $[A / B / C]_{r}$ type laminate with aspect ratio of $a / b=1(a=b=1 \mathrm{~m})$ is considered.

For simplicity, it is assumed that $A=0^{\circ}, B=90^{\circ}$ and $r=1$. Since one of the few efficient analytical solutions of the specially orthotropic plate is Navier solution, and this is good for the case of the four edges simple supported, F.D.M. is used to solve this problem and the result is compared with the Navier solution.

Calculation is carried out with different mesh sizes and the maximum errors at the center of the plate are as follows.

mesh size $10 \times 10$ : error $0.140 \%$

mesh size $20 \times 20$ : error $0.035 \%$

mesh size $40 \times 40$ : error $0.009 \%$

The error is less than $1 \%$. This is smaller than the predicted theoretical errors;

If F.D.M is applied to these equations, the resulting matrix equation is very large in sizes, but the tridiagonal matrix calculation scheme used by Kim \& Han is very efficient to solve such equations [4-7].

Since one of the few efficient analytical solutions of the specially orthotropic plate is Navier solution, and this is good for the case of the four edges simple supported, F.D.M is used to solve this problem. The result is satisfactory as expected.

By neglecting the $M_{x}$ terms, the sizes of the matrices needed to solve the resulting linear equations are reduced to two thirds of the "non-modified" equations.

\section{NUMERICAL EXAMINATION}

\subsection{Bridge deck structures}

The bridge deck structure under consideration is as shown in Fig. 1 and Fig. 2.

$$
\begin{array}{ll}
E_{s}=200,000 \mathrm{MPa}, v=0.3 \\
\text { - Girder - } & \text { - Cross beam - } \\
H_{L}=500 \mathrm{~mm} & H_{T}=300 \mathrm{~mm} \\
B_{L}=200 \mathrm{~mm} & B_{T}=150 \mathrm{~mm} \\
T_{1_{L}}=15 \mathrm{~mm} & T_{1_{T}}=10 \mathrm{~mm} \\
T_{2_{L}}=20 \mathrm{~mm} & T_{2_{T}}=18.5 \mathrm{~mm}
\end{array}
$$

The stiffnesses are given in Table 1 . Type 1 is for the specially orthotropic plate and Type 2 is the case of a simple beam. In oder to a study the effect of the cross beam sizes, variable values of $D_{22}$ are given, in Table 2 .

Analysis is carried out and the result is given by Tables from 3 to 6 . As Table 3 shows, the deflection of Type 2, based on beam theory, is 2.43 times that of specially orthotropic theory.

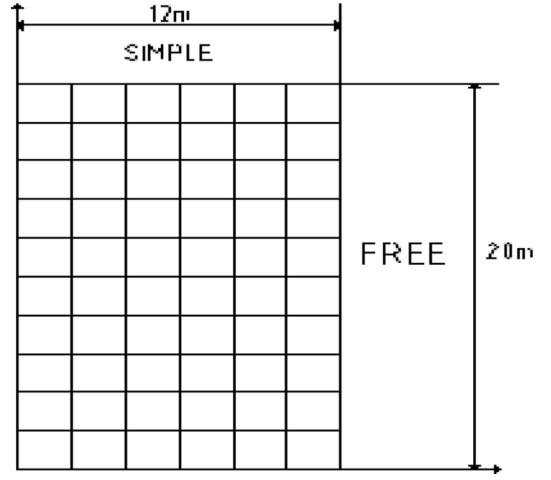

Fig. 1. Bridge deck structure under consideration

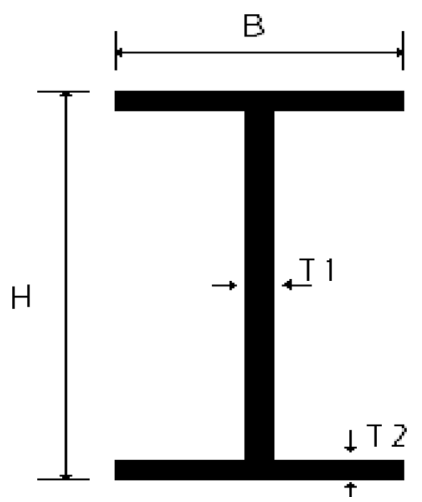

Fig. 2. Cross section of $\mathrm{H}$ beam

Table 1. Stiffnesses

\begin{tabular}{crr}
\hline$D_{i j}(\mathrm{~N} \cdot \mathrm{m})$ & Type 1 & \multicolumn{1}{c}{ Type 2 } \\
\hline$D_{11}$ & 101199927.65 & 101199927.65 \\
$D_{22}$ & 21757837.94 & 0.00 \\
\hline
\end{tabular}

Table 2. Stiffnesses with variable $D_{22}$

\begin{tabular}{cccc}
\hline$D_{i j}(\mathrm{~N} \cdot \mathrm{m})$ & Case 1 & Case 2 & Case 3 \\
\hline$D_{11}$ & 101199927.6 & 101199927.6 & 101199927.6 \\
$D_{22}$ & 21757837.94 & 41618360.36 & 61478882.78 \\
\hline
\end{tabular}

Table 3. Deflection at the center $(\mathrm{m})$ Loading : $100 \mathrm{kN}$ at the center

\begin{tabular}{cccc}
\hline Type & 1 & 2 & Type2/Type1 \\
\hline$\delta(\mathrm{m})$ & $0.6765 \mathrm{E}-01$ & $0.1646 \mathrm{E}+00$ & 2.43 \\
\hline
\end{tabular}

Table 4. Deflection at the center $(\mathrm{m})$ Loading : $100 \mathrm{kN}$ at the center

\begin{tabular}{cccccc}
\hline Case & 1 & 2 & 3 & 4 & 5 \\
\hline \multirow{2}{*}{$\delta(\mathrm{m})$} & 0.6765 & 0.6262 & 0.6061 & 0.5951 & 0.5881 \\
& $\mathrm{E}-01$ & $\mathrm{E}-01$ & $\mathrm{E}-01$ & $\mathrm{E}-01$ & $\mathrm{E}-01$ \\
\hline Case i/Case 1 & 1.0 & 1.0803 & 1.1162 & 1.1368 & 1.1503 \\
\hline
\end{tabular}


Table 5. Natural Frequency (rad/sec) Loading : $100 \mathrm{kN}$ at the center

\begin{tabular}{|c|c|c|c|c|c|}
\hline Type & \multicolumn{2}{|l|}{1} & 2 & \multicolumn{2}{|c|}{$\begin{array}{l}\text { Type2/ } \\
\text { Type1 }\end{array}$} \\
\hline$w(\mathrm{rad} / \mathrm{sec})$ & $0.7313 \mathrm{E}$ & & $.5133 \mathrm{E}+01$ & & 7019 \\
\hline \multicolumn{6}{|c|}{$\begin{array}{l}\text { Table 6. Natural Frequency }(\mathrm{rad} / \mathrm{sec}) \\
\text { Loading: } 100 \mathrm{kN} \text { at the center }\end{array}$} \\
\hline Case & 1 & 2 & 3 & 4 & 5 \\
\hline$w(\mathrm{rad} / \mathrm{sec})$ & $\begin{array}{c}0.7313 \\
\mathrm{E}+01\end{array}$ & $\begin{array}{c}0.7471 \\
\mathrm{E}+01\end{array}$ & $\begin{array}{c}0.7539 \\
\mathrm{E}+01\end{array}$ & $\begin{array}{c}0.7577 \\
\mathrm{E}+01\end{array}$ & $\begin{array}{c}0.7603 \\
E+01\end{array}$ \\
\hline Case i/Case 1 & 1.0 & 1.0216 & 1.0309 & 1.0361 & 1.0397 \\
\hline
\end{tabular}

Increase of the cross beam sizes does not produce profound change of deflection, Table 4.

Similar conclusion can obtained from the frequency, Table 5 and 6. The specially orthotropic plate theory yields much stiffer structure than by beam theory.

\subsection{Influence of the aspect ratio}

The plate considered is as shown in Fig. 3.

The material properties are:

$E_{1}=67.36 \mathrm{GPa}, E_{2}=8.12 \mathrm{GPa}$

$G_{12}=3.0217 \mathrm{GPa}$

$v_{12}=0.272, v_{21}=0.0328, r=1$

$t_{1}=t_{2}=t_{3}=0.005 \mathrm{~m}$

Fiber orientation $\left[0^{\circ} / 90^{\circ} / 0^{\circ}\right]$

The stiffnesses are:

$D_{11}=2,927 \mathrm{~N} \cdot \mathrm{m}, D_{22}=18,492 \mathrm{~N} \cdot \mathrm{m}, D_{12}=627 \mathrm{~N} \cdot \mathrm{m}$

$a=n b,(n:$ an integer 1 5)

and $D_{66}=849, b=3 \mathrm{~m}$

Transverse loading on the plate $: q=286.65 \mathrm{~N} / \mathrm{m}^{2}$

In order to study the influence of $\mathrm{Mx}$ on the equilibrium equations, three cases are considere:

Case A : $w, M_{x}$ and $M_{y}$ are considered.

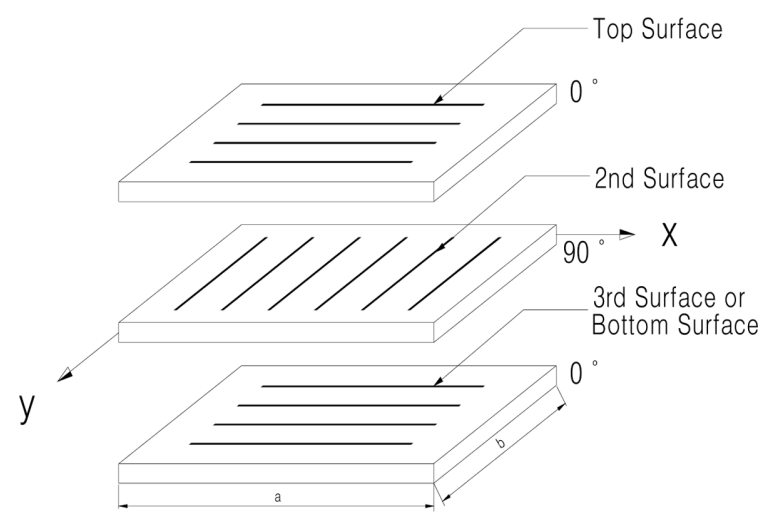

Fig. 3. Configuration of $[a / b / a]_{r}$ Laminated Plate

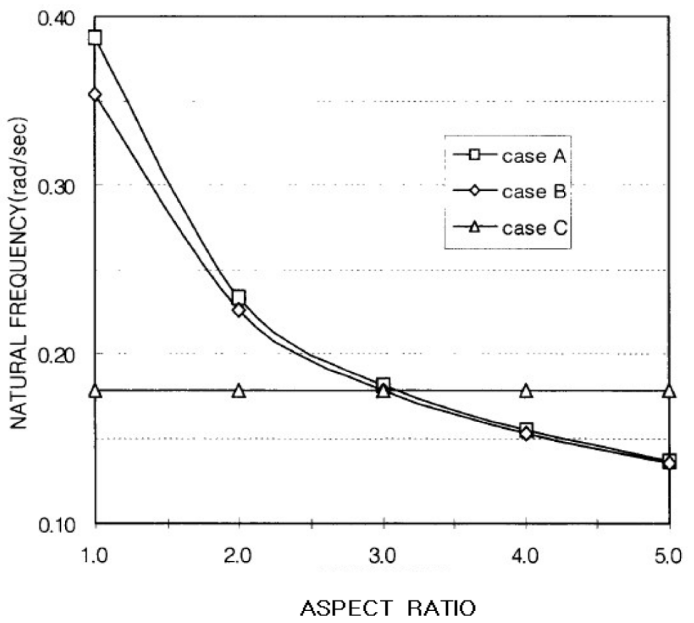

Fig. 4. Natural Frequency of Case A, Case B, Case C (SS)

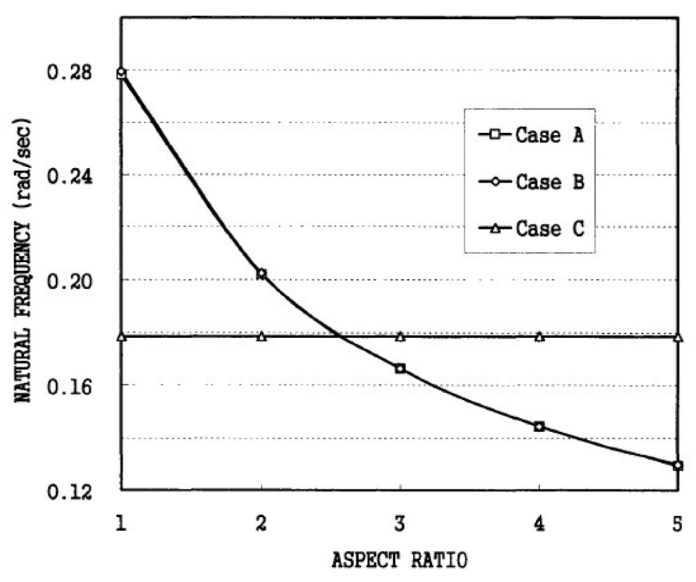

Fig. 5. Natural Frequency of Case A, Case B, Case C (SF)

Case B : $w$ and $M_{y}$ are considered, $M_{x}$ is neglected. Case C : Beam with unit width.

F.D.M. is used to obtain $w, M_{x}$ and $M_{y}$, and obtain the natural frequency. The result is as shown in Fig. 4 and 5. Plates with all edges simple supported (SS), the aspect ratio and the natural frequencies at the center of the uniformly loaded plate are as shown in Fig. 4.

Plates with one side simple and the other side free supported $(\mathrm{SF})$, the aspect ratio and the natural frequencies at the center of the uniformly loaded plate are as shown in Fig. 5.

It is concluded that, for all boundary conditions, neglecting $M_{x}$ terms is acceptable if the aspect ratio $(a / b)$ is equal to or larger than 2 .

\section{CONCLUSION}

For practical design purposes, it is desirable to simplify the vibration analysis procedure. One of the methods is to neglect the influence of the longitudinal moment terms. This paper aims to give some guideline and the way to apply the advanced 
composite materials theory to the structures to the practicing engineers.

A natural frequency of a structure is the frequency under which the deflected mode shape corresponding to this frequency begins to diverge under the resonance condition. From the deflection caused by the free vibration, the force required to make this deflection can be found and from this force, the resulting deflection can be obtained.

Theories for composite material structures are too difficult for such design engineers for construction and some simple but accurate enough methods are necessary. The simply supported laminated plates are analyzed by the specially orthotropic laminates theory. In this paper. the influence of the aspect ratio on the specially orthotropic laminated plates is studied and it is concluded that the method used is sufficiently accurate for engineering purposes.

Most of the bridge deck structures have plate aspect ratios larger than 2. For such cases, design analysis becomes much simpler if influence of the longitudinal moment terms on the relevant differential equations of equilibrium can be neglected. The result of the study on this subject is presented in this paper.

It is concluded that, for all boundary conditions, neglecting the longitudinal moment terms are acceptable if the aspect ratio is equal to or larger than 2 . The result of this paper can be used for simply supported laminated plates analysis.

\section{ACKNOWLEDGEMENT}

This study was partially supported by Seoul National University of Science and Technology.

\section{REFERENCES}

1. Kim, D.H., "Simple Method of Analysis for Preliminary Design of Certain Composite Laminated Primary for Civil Construc- tion II," Journal of Material Technology, Elsevier, London, 1993.

2. Kim, D.H., "Composite Structures for Civil and Architectural Engineering," E\&FN SPON, Chapman \& Hall, London, 1995.

3. Goldberg, John E., and Kim, D.H., "The Effect of Neglecting the Radial Moment Term in Analyzing a Sectorial Plate by Means of Finite Differences", Proc. of the Seventh International Symposium on Space Technolog and Sciences, Tokyo, Japan, 1967.

4. Han, B.K., and Kim, D.H., "Simple Method of Vibration Analysis of Three Span Continuous Reinforced Concrete Bridge with Elastic Intermediate Supportm," Journal of Korean Society for Composite Materials, Vol. 17, No. 3, 2004, pp. 23-28.

5. Han, B.K., and Kim, D.H., "Analysis of Steel Bridges by Means of Specially Orthotropic Plate Theory," Journal of Korean Society of Steel Construction, Vol. 13, No. 1, 2001, pp. 61-69.

6. Han, B.K., and Kim, D.H, "A Study on Size/Scale Effects in the Failure of Specially Orthotropic Slab Bridges," Journal of Korean Society for Composite Materials, Vol. 23, No. 1, 2010, pp. 23-30.

7. Kim, D.H., Han, B.K., Lee, J.H., and Hong, C.W., "Simple Methods of Vibration Analysis of Three Span Continuous Reinforced Concrete Bridge with Elastic Intermediate Supports," Proceeding of the Advances in Structural Engineering and Mechanics, Seoul, Vol. 2, pp. 1279-1284, 1999.

8. Kim, D.H., "A Method of Vibration Analysis of Irreqularly Shaped Structural Members", Proceedings, International Symposium on Engineering Problems in Creating Coastal Industrial Sites, Seoul, Korea, October, 1974.

9. Stephen P. Timoshenko, and S. Woinowsky-krieger, "Theory of Plates and Shells, Second Edition", Mcgraw Hill Book Co, 1989.

10. Ashton, J.E., "Anisotropic Plate Analysis-Boundary Condition", Journal of Composite Materials, pp. 162-171, April, 1970.

11. Whitney, J.M., and Leissa, A.W., "Analysis of a Simply Supported Laminated Anisotropic Rectangular Plate," Journal of AIAA, Vol. 8, No. 1 pp. 28-33, 1970.

12. Pagano, N.J., "Exact Solution for Rectangular Bidirectional Composites and Sandwich Plates," Journal of Composites Materials, Vol. 4, No. 1, pp 20-34, Jan, 1970. 